\title{
BMJ Open Clinical efficacy of COMPASS, a digital cognitive-behavioural therapy programme for treating anxiety and depression in patients with long-term physical health conditions: a protocol for randomised controlled trial
}

Katrin Hulme (D) , Joanna L Hudson, Federica Picariello (D) , Natasha Seaton, Sam Norton, Abigail Wroe, Rona Moss-Morris

To cite: Hulme K, Hudson JL, Picariello $\mathrm{F}$, et al. Clinical efficacy of COMPASS, a digital cognitive-behavioural therapy programme for treating anxiety and depression in patients with long-term physical health conditions: a protocol for randomised controlled trial. BMJ Open 2021;11:e053971. doi:10.1136/ bmjopen-2021-053971

- Prepublication history and additional supplemental material for this paper are available online. To view these files, please visit the journal online (http://dx.doi.org/10.1136/ bmjopen-2021-053971).

$\mathrm{KH}$ and $\mathrm{JLH}$ are joint first authors.

Received 01 June 2021 Accepted 04 October 2021

\section{Check for updates}

(C) Author(s) (or their employer(s)) 2021. Re-use permitted under CC BY-NC. No commercial re-use. See rights and permissions. Published by BMJ.

Health Psychology Section, Psychology Department, King's College London, London, UK

Correspondence to

Rona Moss-Morris;

rona.moss-morris@kcl.ac.uk

\section{ABSTRACT}

Introduction Approximately $30 \%$ of people with longterm physical health conditions (LTCs) experience mental health problems, with negative consequences and costs for individuals and healthcare services. Access to psychological treatment is scarce and, when available, often focuses on treating primary mental health problems rather than illness-related anxiety/depression. The aim of this study is to evaluate the clinical efficacy of a newly developed, therapist-supported, digital cognitivebehavioural treatment (COMPASS) for reducing LTC-related psychological distress (anxiety/depression), compared with standard charity support (SCS).

Methods and analysis A two-arm, parallel-group randomised controlled trial (1:1 ratio) with nested qualitative study will be conducted. Two-hundred adults with LTCrelated anxiety and depression will be recruited through national LTC charities. They will be randomly allocated to receive COMPASS or SCS only. An independent administrator will use Qualtrics randomiser for treatment allocation, to ensure allocation concealment. Participants will access treatment from home over 10 weeks. The COMPASS group will have access to the digital programme and six therapist contacts: one welcome message and five fortnightly phone calls. Data will be collected online at baseline, 6 weeks and 12 weeks post-randomisation for primary outcome (Patient Health Questionnaire Anxiety and Depression Scale) and secondary outcomes (anxiety, depression, daily functioning, COVID-19-related distress, illness-related distress, quality of life, knowledge and confidence for illness self-management, symptom severity and improvement). Analyses will be conducted following the intention-to-treat principle by a data analyst blinded to treatment allocation. A purposively sampled group of COMPASS participants and therapists will be interviewed. Interviews will be thematically analysed. Ethics and dissemination The study is approved by King's College London's Psychiatry, Nursing and Midwifery Research Ethics Subcommittee (reference: LRS-19/20 20347). All participants will provide informed consent to take part if eligible. Findings will be published in peerreviewed journals and presented at conferences.
Strengths and limitations of the study

- This study will be the first to use a robust randomised controlled trial design to evaluate the efficacy of a new digital intervention (COMPASS) designed to treat distress (anxiety/depression) in the context of long-term physical health conditions (LTCS).

- The novel recruitment method and centralised hub delivery model enables national, geographic reach for participants.

- An important limitation of the study is that LTC charities offer a variety of support services, so standardisation of the control arm is not possible.

- As part of the COMPASS treatment, participants are asked to complete mood measures for therapeutic feedback at two points in the programme and, although these are not used for trial data, the extra measurement in the one arm may be a confounding factor.

- Another limitation is that participants are selfselected volunteers.

Trial registration number NCT04535778.

\section{INTRODUCTION}

It is estimated that 15.4 million people in England are living with one or more longterm physical health conditions (LTCs), for example, diabetes, chronic obstructive pulmonary disease, multiple sclerosis and asthma. Thirty per cent of individuals with at least one LTC also have a comorbid mental health condition, which translates to 4.6 million people in England alone. ${ }^{1}$

The presence of a comorbid mental health condition is estimated to increase physical healthcare costs by $45 \%-75 \%$, equating to an extra £1760-£2933 per patient per year. 
This translates to an extra £7.9-£13.2 billion per year to the NHS. LTCs with comorbid mental health conditions also generate wider economic costs, such as increased absence from work and disability leave. ${ }^{2}{ }^{3}$ Treating comorbid depression and anxiety in LTCs should, therefore, improve health outcomes and reduce healthcare costs.

Recent research, including a meta-analysis of therapy outcomes across Improving Access to Psychological Therapy (IAPT) service in England, showed that people with LTCs had poorer outcomes for depression and anxiety than those without an LTC. ${ }^{4}$ The additional complexity of living with an LTC may contribute to the poorer outcomes, or it may be that therapy needs to be tailored specifically for LTCs. ${ }^{5}$ There is some evidence in support of the latter. An analysis of the early roll out of IAPT for people with LTCs found that services who adapted existing mental health protocols to consider the specific challenges of people with LTCs reported greater improvements in mental health outcomes when compared with services that used non-LTC tailored protocols. ${ }^{6}$ Similarly, a randomised controlled trial (RCT) of an online cognitive behavioural therapy (CBT) programme that integrated diabetes and depression treatment needs versus an online CBT programme developed to treat depression only showed larger treatment effects for the tailored diabetes-depression programme. ${ }^{7}$

Moussavi et al highlight the urgency of addressing depression as a public health priority in individuals living with LTCs to reduce disease burden and disability, and to improve the overall health of populations. While a few illness-specific treatment protocols exist like the diabetes example provided above, the authors of this paper are not aware of any evidence-based manualised CBT protocol, which can be used to treat psychological distress across a range of LTCs or where multiple LTCs are present. Where anxiety and/or depression arise as a consequence the LTC, this can be conceptualised as distress resulting from the challenges of adjusting to living with an LTC. ${ }^{9}$

Drawing from the empirical literature, we developed an overarching theoretical model to explain the process of psychological adjustment in LTCs, with the view to outlining treatment mechanisms for LTC distress ${ }^{10}$ (Moon et al, Under review. Treating psychological adjustment in people with physical long-term conditions: an evidence-based theory of adjustment to illness and treatment online). This transdiagnostic model of adjustment to LTCs (TMA-LTC) recognises that having one or more LTCs can result in unique stressors which disrupt a person's emotional equilibrium and require a process of psychological adjustment to regain this. Stressors include critical events such as diagnosis or progression of illness and chronic ongoing stressors such as managing uncertainty and unpleasant symptoms. For each stressor, factors are identified which either hinder or facilitate adjustment. Using this model, we then developed a tailored, interactive, digital CBT treatment programme called 'COMPASS: Navigating your long-term condition', designed specifically around the TMA-LTC (Hulme et $a l$, In prep. The development of COMPASS: a digital health intervention to treat anxiety and depression in the context of long-term physical health conditions). Details of the intervention can be found in the Methods section.

COMPASS was designed to be transdiagnostic, meaning it can be applied to treat psychological distress in the context of any LTC. We chose digital delivery as it can be particularly helpful for people with mobility issues and other healthcare demands that require time and travel. Digital delivery optimises the reach of psychological treatments and removes the need for therapists and patients to be co-located in time and physical space. ${ }^{11} 12$ It overcomes geographical, financial (eg, unpaid leave for healthcare appointments) and other competing barriers to access (eg, healthcare appointments for comorbid health conditions) ${ }^{13-15}$

Online CBT has been shown to have equivalent effectiveness to face-to-face CBT when supported by a therapist. ${ }^{16}$ The importance of therapist support when delivering online CBT treatments is further reinforced by an individual patient meta-analysis, which showed that therapistsupported online CBT has better depression outcomes than unsupported online CBT at posttreatment. ${ }^{17}$

The online delivery of therapy has gained heightened importance in the context of the COVID-19 pandemic. Although the nature of this intervention and trial are not specific to the pandemic, a recent Lancet Psychiatry position paper reported on the negative impact of COVID-19 and identified people with LTCs as a population at increased vulnerability to experiencing poorer mental health outcomes and increased distress, for example, due to delays in treatment and disruption to care. ${ }^{18}$ This was evidenced in the considerable increase in demand on LTC charity support services during this period; for example, National Rheumatoid Arthritis Society reported a $600 \%$ increase in demand for their helpline and Kidney Care UK found that 4 in 10 of the chronic kidney disease population reported worsened mental health since the COVID-19 outbreak ${ }^{19}$ (personal communication, June 2020). This highlights that the timing of this trial may be particularly pertinent given the need for additional support for these populations and the fact that much of mental healthcare in the UK moved to online delivery at the start of the pandemic.

The aim of this RCT is to compare the efficacy of COMPASS, a novel digital CBT programme for adjustment to LTCs, to the well-being and mental health support offered by five LTC charities based in the UK.

\section{Main research question}

Is a therapist-guided, digital CBT programme (COMPASS: navigating your long-term condition) efficacious at reducing distress (Patient Health Questionnaire Anxiety and Depression Scale, PHQ-ADS ${ }^{20}$ ) in people with LTCs when compared with standard charity support (SCS) alone? 


\section{Research objectives}

Primary objective

1. To test the efficacy of COMPASS for treating psychological distress ${ }^{20}$ (primary outcome) in people with LTCs when compared with SCS alone.

We hypothesise that people receiving COMPASS will report significantly greater reductions in psychological distress (PHQ-ADS) compared with SCS alone.

\section{Secondary objectives}

1. To test the efficacy of COMPASS versus SCS alone on self-reported clinical outcomes (secondary outcomes), including depression, anxiety, functioning, quality of life, COVID-19-related distress, illness-related distress, knowledge and confidence of illness self-management, and perceived LTC symptom severity and improvement.

2. To assess the cost effectiveness of COMPASS versus SCS alone on costs and quality-adjusted life years (secondary paper).

3. To explore factors impeding and facilitating COMPASS use by patients and therapists.

We hypothesise that COMPASS will demonstrate greater improvements in secondary outcomes compared with SCS alone.

\section{METHODS AND ANALYSIS \\ Design}

This study is a two-arm, parallel-group RCT with a nested qualitative study. We aim to recruit 200 people with LTCs experiencing illness-related distress (anxiety and/ or depression) who will be individually randomised to receive COMPASS or SCS alone, for 10 weeks. Participants have a 50:50 chance of being allocated to receive COMPASS or SCS alone. Data, including the primary outcome: PHQ-ADS, ${ }^{20}$ will be collected online at baseline, mid-treatment ( 6 weeks post-randomisation) and end-oftreatment (12 weeks post-randomisation). Some participants will also be invited to take part in interview(s) to talk about their expectations (prior to) and experiences of (post) COMPASS.

As per guidance outlined by the PRECIS-2 tool, this trial adopts an explanatory approach, as opposed to 'real life' pragmatic one, by exploring whether the treatment can work under controlled conditions, that is, evaluating efficacy. ${ }^{21}$ However, as the authors note, the explanatory and pragmatic design approaches are not dichotomous or mutually exclusive. ${ }^{21}$ Therefore, several elements of this trial, for example, the control arm (charities' support services) and recruitment, mean that it lies close to half-way on the continuum between the two, illustrated in the scores depicted in figure 1.

\section{Setting}

Due to the COVID-19 pandemic, setting up research studies in the NHS is prohibited, unless they are COVID related. Therefore, participants will be recruited through five national LTC charities based in the UK: Crohn's and

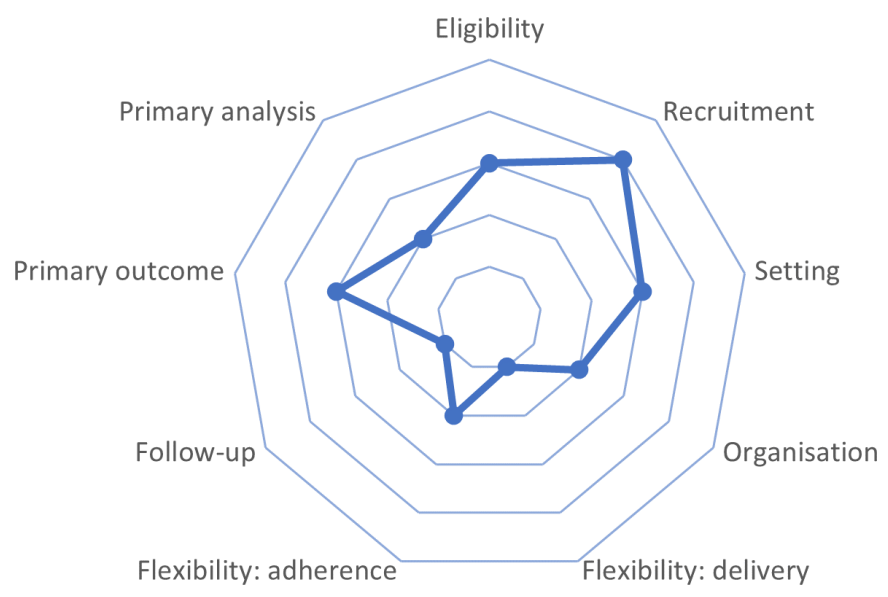

Figure 1 Radar plot of PRECIS-2 factors to determine the type of approach taken, rated from 1 (very explanatory) to 5 (very pragmatic). ${ }^{21}$

Colitis UK, Kidney Care UK, MS Society, Shift.ms and Psoriasis Association. The treatment will be delivered at participants' homes via the internet and telephone calls. Therapists will deliver the telephone and messaging support remotely from their place of work or home (if working from home during the COVID-19 pandemic).

\section{Target population}

Inclusion criteria

1. Aged $\geq 18$ years.

2. Verbal and written proficiency in English

3. UK resident (GP registered)

4. Basic computer literacy.

5. Email address to register with COMPASS.

6. Clinical levels of depression or anxiety: a symptom score of $\geq 3$ on the depression or anxiety items of PHQ$4 .^{22}$

7. Self-reported diagnosis of long-term condition, confirmed by patient providing details of diagnosis (eg, LTC, subtype where applicable, when diagnosed, confirmation that diagnosis was made by qualified medical practitioner).

8. Distress experienced is related to a long-term condition (ie, answer 'Yes' or 'Sometimes' to Q8 in eligibility questionnaire (see online supplemental appendix 1)).

\section{Exclusion criteria}

1. Self-report of existing substance dependency, moderate to severe cognitive impairment and severe mental health conditions (eg, psychosis).

2. Suicidal risk: identified by 'Yes' to risk question (are you currently making plans about how you would end your life?). ${ }^{22}$

3. Currently receiving psychological treatment from psychologist/counsellor/therapist or online psychological treatment.

Withdrawal criteria

Participants will be withdrawn from the trial if a psychological or disease-specific issue is identified that requires 
immediate onward referral meaning that the participant is not suitable for COMPASS or cannot comply with study procedures, and an adverse event which requires discontinuation of the study or results in the inability to comply with study procedures.

Participants are also able to withdraw if they choose, without giving a reason. If in the COMPASS arm, participants will be able to choose to 'drop-out' from the treatment only (ie, stop doing COMPASS but continue to do questionnaires) or 'withdraw' from all further aspects of the research trial. If in the COMPASS arm, participants will be asked to confirm whether they are happy to continue with the research questionnaires and/or follow-up interview. Reasons for drop-out and withdrawal will be recorded if provided by participants.

\section{Randomisation}

Randomisation will occur at the individual level using a 1:1 allocation ratio, stratified by healthcare condition to ensure a balance in the number of participants with different LTCs across the treatment and control arms. Qualtrics' built-in randomiser will be used for random allocation. This will be implemented by an administrator independent of the trial team to ensure allocation concealment. A separate Qualtrics account will be used to ensure blinding of the data analyst and group allocation will be kept separate from questionnaire data.

\section{CONSORT diagram}

The flow of recruitment though the study will be reported according to Consolidated Standards of Reporting Trials (CONSORT) guidance. ${ }^{23}$ Study processes (including recruitment, eligibility screening and follow-up) and the number of participants at each stage will be presented in a CONSORT diagram, as shown in figure 2.

\section{Blinding}

All researchers involved in follow-up quantitative data collection and statistical analyses will remain blinded to treatment allocation, including the trial data analyst. Data files containing participant allocation information, used to notify participants of their group, record when treatment is complete and contact for interview, will be password protected so that they are inaccessible to the blinded individuals. Statistician and data analyst will always be blinded unless unblinding incidents happen during follow-up data collection facilitated by the data analyst where participants inadvertently reveal their allocation. A record of unblinding incidents will be maintained throughout the duration of the trial.

As this is a therapeutic treatment trial, it is not possible to blind the participants or the therapists.

\section{Planned treatment}

There is one experimental treatment being assessed in this study. COMPASS is a digital, therapist-supported, CBT-based programme designed to treat illness-related distress in the context of physical LTCs. It is CE marked as a class I medical device, in compliance with the Medical

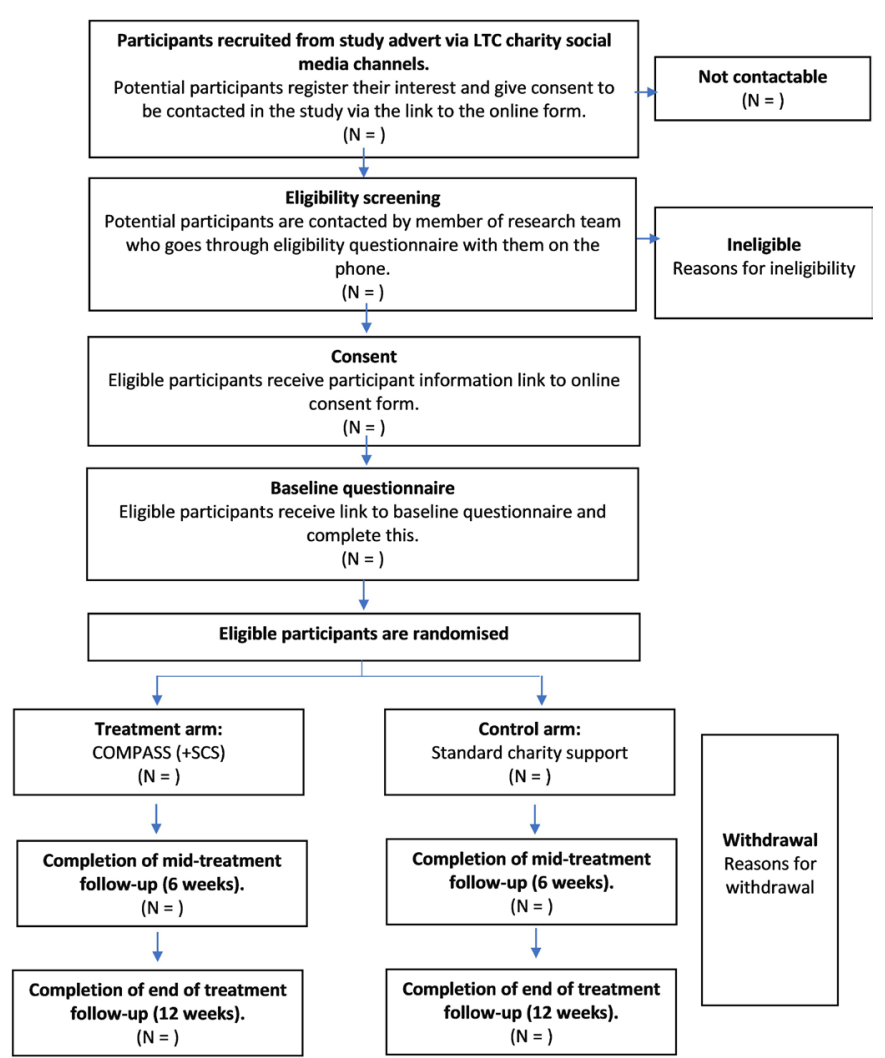

Figure 2 CONSORT diagram indicating study recruitment, eligibility screening and follow-up. CONSORT, Consolidated Standards of Reporting Trials; LTC, long-term physical health condition; SCS, standard charity support.

Devices Directive 93/42/EEC, and consists of a webbased platform for patients and a platform for therapists (Hulme et al, in prep).

It consists of 11 modules, which target evidence-based mechanisms of action that trigger and sustain psychological distress in LTCs, for example, managing uncertainty, managing symptoms (Moon et al, under review). A detailed overview of sessions, targeted factors and interactive/therapeutic tasks is presented in table 1 . The first module, Navigating Compass, is compulsory and includes interactive self-assessment tasks, which lead to a tailored and personal formulation of the key problem areas. The patient uses this information to choose their journey through the COMPASS programme. The remaining sessions, organised across the four compass quadrants, can be completed in any order the patient would like, depending on the things they would like to focus on. The sessions use psychoeducation, patient stories, goal setting and interactive tasks based on evidence-based CBT techniques. Patients are also encouraged to reflect on module content and their progress. At regular intervals, patients are prompted to complete mood measures (PHQ-9: depression, seven-item Generalised Anxiety Disorder Scale (GAD-7): anxiety) and scores are graphically represented for them to see.

Table 1 outlines the COMPASS modules, the respective adjustment factors they address and the evidence-based CBT techniques that guide the content. 
Table 1 Overview of COMPASS modules, the content and the cognitive behavioural therapy techniques used

\section{Evidence-based cognitive}

COMPASS module Adjustment factors and cognitive-behavioural behavioural therapy techniques Interactive tasks patient name

mechanisms targeted

used

completes

Core module that patients complete before accessing the other modules

Navigating Compass Introduction to cognitive-behavioural processes

1. Mapping my LTC associated with anxiety/depression in LTCs for patient to identify ones relevant to them

- Assessment

Personal five area model

2. Key skills

Feelings of helplessness and hopelessness because of LTC self-management demands

- Formulation

- Guided discovery

- Problem solving

Problem solving

Goal setting

Goal setting

\section{COMPASS quadrant: north - navigating change and uncertainty}

Managing uncertainty Uncertainty linked to LTC symptoms and health/ social outcomes.

Problem solving

- Balancing illness selfmanagement skills with emotionfocused coping

$\begin{array}{lll}\text { Power of thoughts } & \begin{array}{l}\text { Unhelpful or inaccurate beliefs about symptoms, Cognitive restructuring Identifying thoughts } \\ \text { illness and treatments. } \\ \text { Increase cognitive flexibility }\end{array} & \text { Types of unhelpful } \\ \text { thoughts } & \text { Challenging thoughts }\end{array}$

COMPASS quadrant: east-even keel

\begin{tabular}{|c|c|c|c|}
\hline Achieving routine & $\begin{array}{l}\text { Inconsistent 'boom-bust' patterns of activity } \\
\text { whereby a person pushes to do things which } \\
\text { then results in them crashing }\end{array}$ & $\begin{array}{l}\text { - Planning patterns of activity and } \\
\text { rest } \\
\text { Self-monitoring }\end{array}$ & - Activity diary \\
\hline Managing symptoms & $\begin{array}{l}\text { Focusing on symptoms and coping in an } \\
\text { unhelpful way }\end{array}$ & $\begin{array}{l}\text { Attentional techniques } \\
\text { Self-monitoring: symptom } \\
\text { records } \\
\text { Cognitive restructuring } \\
\text { - Relaxation }\end{array}$ & $\begin{array}{l}\text { Symptom re-think } \\
\text { Personal fatigue/pain } \\
\text { model }\end{array}$ \\
\hline Emotions & $\begin{array}{l}\text { Normalising distress. } \\
\text { Appropriate expression and acknowledgement } \\
\text { of emotions }\end{array}$ & $\begin{array}{l}\text { Normalising } \\
\text { Behavioural activation } \\
\text { Acceptance } \\
\text { - Emotional expression }\end{array}$ & $\begin{array}{l}\text { Personal emotions model } \\
\text { (distress) } \\
\text { Feel good model }\end{array}$ \\
\hline
\end{tabular}

COMPASS quadrant: south-support

\begin{tabular}{llll}
$\begin{array}{l}\text { Strengthening } \\
\text { personal } \\
\text { relationships }\end{array}$ & Social support from others (helpful and unhelpful) & Assertive communication & Smotional expression \\
$\begin{array}{l}\text { Making use of } \\
\text { professional support } \\
\text { and information }\end{array}$ & $\begin{array}{l}\text { Relationship with healthcare professionals. } \\
\text { Helplessness around illness self-management }\end{array}$ & $\begin{array}{l}\text { Assertive communication } \\
\text { Signposting and care } \\
\text { coordination }\end{array}$ & Increasing assertiveness \\
\hline
\end{tabular}

COMPASS quadrant: west-living well

\begin{tabular}{|c|c|c|c|}
\hline Healthy lifestyle & Encouraging positive health behaviours & $\begin{array}{l}\text { Cross-condition interventions: } \\
\text { identify and manage general health } \\
\text { behaviour problems: } \\
\text { Nutrition } \\
\text { Sleep } \\
\text { Stress } \\
\text { Medication adherence }\end{array}$ & \\
\hline I am me, not my LTC & $\begin{array}{l}\text { Self-compassion } \\
\text { Self-efficacy } \\
\text { Self-esteem }\end{array}$ & $\begin{array}{l}\text { Compass-focused intervention } \\
\text { Motivational interviewing } \\
\text { Cognitive restructuring }\end{array}$ & $\begin{array}{l}\text { Self-compassion task } \\
\text { Problem solving: self- } \\
\text { efficacy } \\
\text { Increasing self-esteem }\end{array}$ \\
\hline Managing stress & $\begin{array}{l}\text { Normalising stress. } \\
\text { Helpful coping strategies }\end{array}$ & $\begin{array}{l}\text { Proactive self-management skills } \\
\text { Relaxation }\end{array}$ & - Relaxation exercises \\
\hline
\end{tabular}

LTC, long-term physical health condition.

Participants allocated to COMPASS will be notified by email and will be registered on the online platform by a member of the research team. They will then receive an automated email from the COMPASS programme, providing them with their login details (including temporary password) and prompting them to $\log$ in to complete the registration process. Their therapist sends them a welcome message containing information about the programme, recommending regular logins and confirming the first phonecallappointment. Thirty-minute 
phone calls (or therapeutic online messages if preferred) are then scheduled fortnightly for a period of 10 weeks, that is, welcome message in week 1 and calls/messages in week 2 , week 4 , week 6 , week 8 and week 10 . In week 4 and week 8, patients will be sent questionnaires (PHQ-9, GAD-7 and Work and Social Adjustment Scale (WSAS)) via the COMPASS programme, to provide therapists and patients with an overview of their mood and progress. This information is used to provide feedback on progress (or lack of it) to therapists and patients, consistent with principles of case management. ${ }^{24}$ Questionnaires are recognised as part of good therapeutic practice, allowing monitoring of therapy for patients, therapists and supervisors as a way of providing feedback of what is working well, making adjustments where needed and identifying safety and risk issues. Therefore, the questionnaires are deemed as a part of the intervention package.

An important consideration is that the imbalance in the number of administrations of the assessments used across the COMPASS and control arm may introduce bias in the efficacy estimate due to re-test artefacts, although this is likely to be small. ${ }^{25}$

\section{Existing evidence}

COMPASS has been implemented in several south-east London NHS services to test the feasibility of using the programme in current routine care. Data collected from January 2019 to June $2020 \quad(\mathrm{n}=74)$ demonstrated significant improvements in anxiety, depression, combined anxiety/depression (distress) and functioning measures (Seaton $e t$ al, Under review. Recovery in long-term condition patients: an analysis of an IAPT service). Findings from this work informed improvements to the programme, for example, reduction in text and improved appointment scheduling functionality. These will be completed prior to the launch of this current study. Additionally, findings informed the planning of the current study:

- In the implementation feasibility work, the primary outcomes were anxiety and depression, in line with IAPT minimum data set outcomes. ${ }^{26}$ However, research suggests that it may be useful to conceptually distinguish between primary mental health disorders and illness distress. ${ }^{9}$ Therefore, the primary outcome for this study will be the combined depression/anxiety score.

- In the implementation feasibility work, therapist support was initially provided primarily via in-site messages. However, patient engagement improved once telephone support was the default support option. Therefore, this was the primary communication mode for this study, in line with similar trials. ${ }^{27} 28$

\section{Therapists}

COMPASS is a guided treatment programme with support provided at regular intervals throughout the programme by trained therapists, called 'guides'. Therapists will be clinical/health psychology trainees or qualified clinical or health psychologists. They will be CBT trained.
Therapists will have six review sessions with each patient. This consists of a welcome message via the in-site messaging system in week 1 and five fortnightly phone calls in week 2 , week 4 , week 6 , week 8 and week 10. The welcome message outlines expectations for the programme and support calls, reflects on progress made so far and confirms the time and date for the first phone call appointment. The five subsequent phone calls will be of $30 \mathrm{~min}$ each, followed by a summary message.

The content will be guided by the patient and their experiences but broadly speaking will be structured in the following way:

1. Introduction and agenda setting.

2. Explore and discuss issues the patient raises.

3. Review progress, for example, discussing goals, tasks and reflections.

4. Plan for the next 2 weeks, for example, encourage logging in.

If a patient does not attend (DNA) an appointment, the therapist will send a message on COMPASS and a member of the research team will also email the patient in case they are having technical difficulties accessing the programme. If possible, the therapist will try to reschedule the appointment. If this is not possible, the patient will be informed of the next scheduled phone call.

\section{Therapist platform}

As well as the patient platform, COMPASS also includes a platform for therapists, which allows them to monitor and review their patients' progress and homework tasks. This platform consists of:

- A caseload management landing page

- This page lists all patients on the caseload according to case-management principles, highlighting actions required.

- A calendar

- Individual patient page:

- This displays patient information, including contact information, progress through the online modules, goals, completed tasks, reflections and self-reported clinical outcomes (completed at registration, at week 4 and week 8 of the treatment, and at discharge).

- Messaging system:

- This allows therapist and patient to send and receive messages via the COMPASS platform.

- Appointment system:

- This allows therapist to schedule and record their clinical contacts with patients, including:

- Dates and time of appointment.

- Attendance

- Duration.

- Purpose of appointment.

- Mode of support.

- Outcome (continue, step-up, discharge and other).

- Notes. 


\section{Therapist training}

Each therapist will receive training in (a) the transdiagnostic LTC adjustment theory underlying the COMPASS content and therapeutic delivery and (b) the technical aspects of COMPASS and research procedures. The two sessions will each take 2-3hours. AW (clinical health psychologist) will deliver the LTC therapeutic training session. KH (COMPASS development lead) will deliver the technical/research training. Therapists will be given dummy accounts on the COMPASS testing site to familiarise themselves with the programme, from both patient and therapist perspectives.

The clinical-based, LTC-CBT training consists of information and experiential learning around the models of coping and adjustment, and the bidirectional relationship between distress and physical health symptoms. A variety of learning styles will be used to support therapists in consolidating skills and competences of tailoring CBT techniques to address LTC-specific challenges. These skills include (a) using validation, (b) using curious/open questioning to engage with individuals to understand their presenting issues, critical incidences and ongoing illness stressors and (c) identifying illness specific thoughts and behaviours, and helpful and unintentional consequences of these. Therapists are taught to reflect on the patient's use of COMPASS and explore this together in planning next steps on their COMPASS journey.

Therapists will also take part in 30-minute peer learning sessions, where they present and learn about the presenting LTCs, that is, prognosis, symptoms, possible impact and case studies.

\section{Therapist manuals}

Two manuals will supplement the therapist training. One manual contains the content seen by patients. The other provides guidance about important therapeutic considerations for each of the sessions and an overview of technological functionality.

A document pack will also be provided outlining therapy and study procedures. This will include an appointment checklist (so therapists can check they have covered all the key points when reviewing patients' progress) and DNA and risk standard operating procedures. Message templates (for messages the therapists send to patients on COMPASS) will also be provided.

\section{Therapy supervision}

After training, therapists will receive fortnightly supervision with AW, which may be individual or in peer groups. Therapists will have the opportunity to discuss clinical skills, problem areas or individual patients who may present challenges. Each therapist will be expected to prepare for supervision and to bring supervision questions, which may include recordings of sessions (in addition to fidelity checks). The supervisor will review notes and messages prior to supervision meetings.

Therapists will be expected to review each of their clients at some point along the course of their COMPASS journey. Regular supervision will ensure that the quality of therapeutic support is high and consistent, and that therapists adhere to the protocols.

\section{Treatment fidelity}

Regular supervision will help to ensure fidelity to the treatment. In addition, telephone support sessions will be audio-recorded for the purposes of supervision and checking treatment fidelity. A subset of the audiorecordings will be analysed by an independent rater with therapeutic CBT experience. The audio-recordings rated will be the second and fourth calls (if available) for $10 \%-20 \%$ of the therapists' caseload. The patients for which the recordings will be rated will be selected using the random number generator functionality in Excel.

\section{Treatment as usual: SCS}

Participants in both arms will be able to access SCS, with the control arm being SCS alone. This is defined as the care that is usually available to participants via their charity and, if available, usual NHS care. Participants in the control arm were actively signposted to their charity resources via email, including helpline phone number and website address.

The support services each LTC charity provides are listed below.

\section{MS Society}

1. Welfare benefits advice.

2. Disability law service.

3. Physical activity service.

4. Short breaks service.

5. MS nurses (helpline).

6. Befriending.

7. Online forum.

8. Informational support, including applying for support grants, educational informational about MS and email updates.

9. Interactive online module focused on managing fatigue.

10. Living well with MS virtual sessions.

11. Time to chat sessions.

12. Webinar series.

13. Regional support groups.

\section{Kidney Care UK}

1. Support for applying for financial grants.

2. Advocacy officer support.

3. Respite grants for short breaks away and financial support with household, travel and educational costs.

4. Counselling and support service.

5 . Online community support.

6. Helpline.

Shift.ms

1. Buddy network.

2. MS educational and informational films.

3. Online forum. 
4. Personal protective equipment (PPE) for the mind;a course of podcasts around developing mental resilience.

\section{Psoriasis Association}

1. Confidential helpline aiming to provide information, a listening service and signposting to other organisations.

2. Information about psoriasis, symptoms, managing the condition and treatments.

3. Peer-to-peer support via online forum and groups.

Crohn's and Colitis UK

1. Helpline.

2. Online forum.

3. Virtual social events.

4. Local social groups.

5. Information about symptoms, treatments and general well-being.

Participants allocated to SCS only will be notified by email by a member of the research team, signposted to the respective charity support website and provided with their helpline phone number. For Kidney Care UK only, the contact details of participants in the control arm will be securely transferred to a designated member of the charity counselling service, so they can follow-up with the participant.

Once the 12-week questionnaires have been completed, SCS only participants will be sent a PDF of resources based on COMPASS content, signposting to online resources and outlining some basic tools for managing well-being.

\section{Recruitment}

Potential participants will be reached via LTC charities' social media channels. A link will be included in posts on Twitter, Facebook and Instagram, which directs people to an online form (on Qualtrics) containing the participant information sheet (online supplemental appendix 2) and a 'Register your interest' section where people can provide their contact details (name, email address, phone number and LTC charity).

\section{Study procedures}

Following receipt of these contact details, a member of the research team will contact participants to assess their eligibility through a screening phone call. The eligibility questionnaire can be found in online supplemental appendix 1 . If someone cannot be reached by telephone, they will be emailed to schedule a suitable time for the researcher to call back and complete the screening.

Those who are ineligible will be signposted to relevant charity support services. Those who are eligible will be emailed the participant information sheet again by a member of the research team from a study email account, along with a link to the online study consent form (on Qualtrics) (see online supplemental appendix 3). Participants will give their informed consent by agreeing to the statements and providing an online signature. Once consented, participants will be asked to complete a baseline questionnaire (on REDCap) prior to being randomised to receive COMPASS or SCS only. A subsection of those allocated to the COMPASS arm will be interviewed prior to being registered on the programme if they provided additional consent to this. This is to explore how people understand the link between their distress and their LTC(s), prior to starting the treatment and will be reported in a separate publication.

\section{Data collection}

Below we list all the outcomes relevant to this study. Table 2 summarises their schedule of assessment. Specifically, all primary, secondary and health economic outcomes will be assessed at baseline, mid-therapy (6 weeks post-randomisation) and end-of-treatment (12 weeks post-randomisation) collected online using REDCap. Sociodemographic and clinical information will be collected at screening and baseline:

- Screening: name, DOB, contact information, GP details and eligibility question responses (see online supplemental appendices for form).

- Baseline: gender, age, ethnicity, LTC diagnoses, employment status, occupation, education level, marital status, living arrangements, postcode, psychotropic medication prescription/use and receipt of statutory sick pay.

\section{Summary of time points and measures \\ Measures}

Additional information on each of the primary and secondary outcome measures implemented at the time points presented in table 2 is given below.

\section{Primary outcome}

The primary outcome for this trial will be the PHQ-ADS. ${ }^{20}$ This distress scale has a total of 16 items. Specifically, it includes the nine items from the ${ }^{29} \mathrm{PHQ}$ and the seven items from the GAD. ${ }^{30}$ Each item is responded to on a 4-point Likert Scale (0-3). Summed scores for the total scale can range from 0 to 48 , with higher scores indicating higher levels of distress. A score of $\geq 10$ is used to determine the presence of distress with a change of 4 or more points on the scale is considered a minimum clinically important difference. ${ }^{20}$

\section{Secondary outcome measures}

The secondary outcomes for this study are listed below.

\section{Depressive symptoms}

Depressive symptoms will be assessed using the nine-item PHQ (PHQ-9), ${ }^{29}$ which is a subscale of the PHQ-ADS. ${ }^{20}$ The PHQ-9 has a scale range of $0-27$, higher scores indicate increased depressive symptoms.

\section{Anxiety symptoms}

Anxiety symptoms will be assessed using the seven-item GAD Scale (GAD-7) ${ }^{30}$ which is a subscale of the PHQADS. ${ }^{20}$ The GAD-7 has a scale range of $0-21$, higher scores indicate increased anxiety symptoms. 


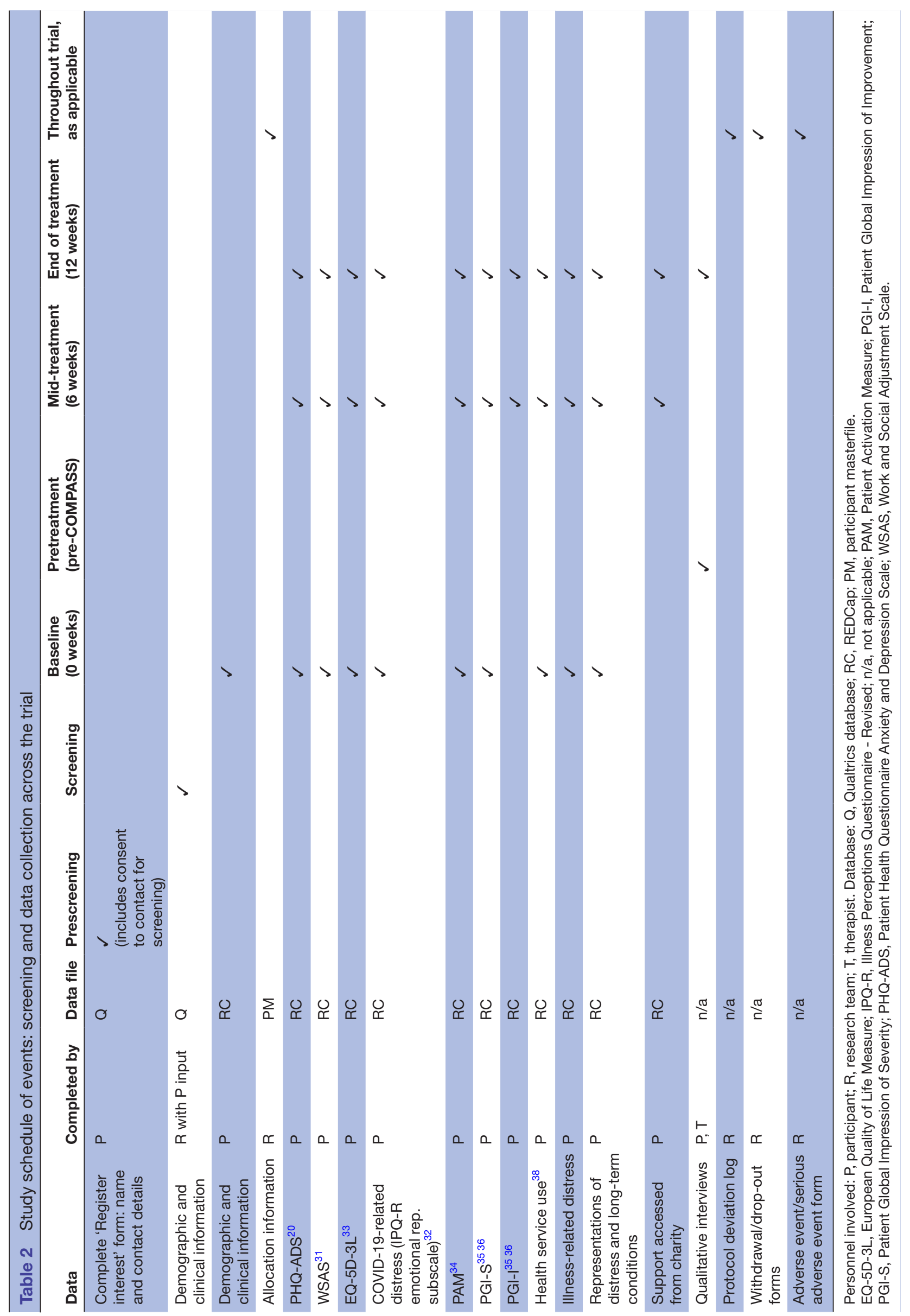

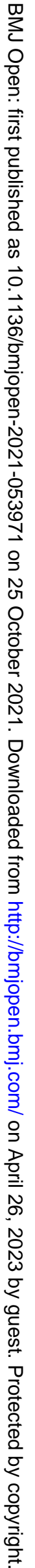




\section{Functioning}

Functioning will be measured by the WSAS, a five-item measure of functional impairment. ${ }^{31}$ The WSAS has a scale range of $0-40$, higher scores indicate greater functional impairment.

\section{COVID-19-related distress}

The emotional representations subscale from the Illness Perceptions Questionnaire-Revised ${ }^{32}$ will be used to assess COVID-19-related distress. The emotional representations subscale consists of six items and assesses a person's emotional response to a specific health conditions/virus, in this case COVID-19. Emotions assessed include low mood, anxiety, fear, worry and anger. Items are responded to on a 5-point Likert scale (1, strongly disagree to 5 , strongly agree). The total score has a range of 6-30. Higher scores indicate greater COVID-19-related distress.

\section{IIIness-related distress}

A bespoke 2-item measure has been developed by the research team to assess illness-related distress. The structure resembles that of the $\mathrm{PHQ}-9^{29}$ and the GAD-7, ${ }^{30}$ whereby the frequency of a distress symptom is indicated using a 4-point scale, ranging from 'not at all' to 'nearly every day'. The items will ask about feelings of distress related to an LTC and feelings of not being able to cope with an LTC. The total score has a range of 0-6. Higher scores indicate greater illness-related distress. Reliability of this scale will be checked using Cronbach's alpha.

\section{Quality of life}

Quality of life will be measured using the European Quality of Life Scale (EQ-5D-3L) ${ }^{33}$ It includes five items (range: 1-3) to assess mobility, self-care, usual activities, pain/discomfort, and anxiety and depression. Higher item scores indicate poorer quality of life. The EQ-5D-3L also includes a visual analogue global health rating (range: 0-100), higher scores indicate better global health ratings.

\section{Knowledge and confidence for illness self-management}

The Patient Activation Measure (PAM) short version will be used to assess knowledge and confidence in illness selfmanagement. ${ }^{34}$ The measure has 13 items. The summed scored on the scale can range from 0 to 100 , with higher scores indicating greater levels of knowledge and confidence in illness self-management.

\section{Long-term physical symptom severity and improvement}

The Patient Global Impression Scales of Severity (PGI-S) and Improvement (PGI-I) (two measures) will be used to assess participants' perceived LTC symptom severity. ${ }^{35} 36$ The PGI-S is a one-item measure, whereby long-term condition severity is ranked on a 4-point scale (range: $0-3$ ). The PGI-I also has one-item measure, where improvement in symptoms is rated on a 7-point scale (range: 0-6). Higher scores indicate greater severity and greater deterioration in the PGI-S and PGI-I respectively.

\section{Additional}

To provide an insight into representations of distress and LTCs and how/if these are linked, participants will be asked to complete the Diabetes and Depression Representation and Management Questionnaire. ${ }^{37}$ This is a 10-item questionnaire, which will be adapted to be transdiagnostic. Each item is answered on a 5-point Likert scale. The questionnaire is made up of three subscales: separate representations (3 items; range: $3-15$ ), negative linked representations (4 items; range: 4-20) and incoherent representations (3 items; range: 3-15). Respectively higher scores indicate greater separation of LTC and distress representations, greater negative relationship between LTC and distress and more incoherent representations of LTC and distress.

To inform the health economic analysis (funding dependent), three health service use items will be included from the Client Service Receipt Inventory ${ }^{38}$ : visited GP, accessed a psychologist/therapist and accessed emergency care in last 3 months.

At mid-treatment and end-of-treatment, all participants will be asked what charity resources they accessed. Charity support will be investigated using a multiple-choice question. Participants will be able to select any combination of the following options (charity helpline, online forums/ networks, phone counselling, information booklets and other) or select 'I have not received any support from the charity'. If other is selected, a free text box appears to describe the support. Participants will be asked to give details of the charity regarding frequency and duration of $\operatorname{contact}(\mathrm{s})$.

Additional survey questions will also be sent to COMPASS users (patients and therapists) after the 12-week questionnaires to collect feedback about the usability and technological aspects of COMPASS. This information will be used to inform tech improvements to the programme for future versions.

\section{Adherence to therapy}

We will also measure adherence using the below data:

1. The number of therapist support sessions attended by participants. This information will be recorded by therapists in COMPASS.

NICE guidance for the management of depression in LTCs recommend a minimum of six therapist support sessions be implemented when treatments are blended with guided self-help resources. ${ }^{39}$ This will be achieved within the timeline of the trial through a welcome message at the beginning and five subsequent support telephone calls.

2. Mean duration of contacts between therapists and participants. This information will be recorded by therapists in COMPASS.

We have suggested that telephone sessions last $30 \mathrm{~min}$ in duration given a previous trial which blended online CBT with therapist support showed that 30-minute phone calls (alongside the online CBT) were effec- 
tive. ${ }^{28}$ Mode of delivery will be recorded by therapists (eg, telephone or in-site message).

3. Adherence to the online modules which a participant completes independently will be automatically recorded by the COMPASS programme. Specifically, the number of online sessions completed by the participant and their mean duration of login.

Patient adherence to COMPASS treatment will be defined as: (a) attended a minimum of three therapist sessions and (b) completed five online COMPASS modules.

It is also important that therapists supporting patients on COMPASS are adherent to the protocol and LTC treatment model. A bespoke treatment fidelity scale will be developed to check whether therapists are using relevant CBT skills. Therapists will audio-record their sessions for reflection in supervision and to be assessed for fidelity.

\section{Proposed sample size}

We aim to randomise 200 participants, split evenly across charities. This provides $80 \%$ power to detect a standardised mean difference, $\mathrm{d}=0.4$, for the PHQ-ADS ${ }^{20}$ (primary outcome) at the $5 \% \alpha$ level (pre-post $=0.4$ ), inflating the sample size for $15 \%$ drop-out, based on rates and weighted average across feasibility studies of similar interventions in people with LTCs. ${ }^{40-43}$ This effect represents a 4-point difference on the PHQ-ADS (SD: 10), which may represent a minimum clinically important difference. ${ }^{20}$

Recruitment is feasible, given calls/contacts to the LTC charities helpline range from 200 to 1500 per month and many more interact with the social media channels of the charities, for example, website, Facebook, Instagram and Twitter. Using a conservative estimate of 200 contacts per month, and assuming 30\% of these people experience symptoms of anxiety and/or depression, ${ }^{1}$ we require only $28 \%$ interacting with the charities who are experiencing distress to participate over a 3-month baseline recruitment period.

We expect to have five therapists each covering $0.5-2$ days/week on the project. Each therapist can see 4-5 patients/day and will have therapeutic contacts with patients fortnightly ( 1 therapist: $8-10$ patients within a 3 -month treatment period). Therefore, 6 days of treatment per week, over a period of 6 months, will be able to treat $96-120$ participants.

\section{Statistical analysis}

Analyses will be conducted following the intention-totreat principle by a data analyst (blind to treatment allocation) with oversight from a senior statistician. All analyses relating to the objectives stated in this protocol will be prespecified in a statistical analysis plan (SAP), which will be finalised and approved by an external trial statistician before data collection is completed.

The data set not containing group allocations for blinded analyses will be provided to the data analyst only after the SAP has been signed off by the chief investigator, senior statistician, data analyst and an independent to the trial statistician. All analyses will be conducted using Stata V.16.1 or higher with the analysis reproducible by saved statistical code.

Means/standards deviations and frequencies with percentages will be used to describe the baseline characteristics of the sample and the post-randomisation outcome measures at each time point by group, and likewise to report measures of adherence to COMPASS treatment.

Treatment effects, as adjusted means between the treatment and control group at each time point, will be estimated using linear mixed-effects models with random effects accounting for repeated observations and partial clustering by therapist. Covariates will include treatment group, time, time-by-group interaction, baseline level of outcome and dummy coded LTC indicator variables. Unstandardised and standardised (Hedge's g) treatment effect estimates will be presented with $95 \%$ CIs.

Sensitivity analyses will be undertaken to explore the impact of assumptions around missing data, clinical levels of distress at baseline, adherence to the treatment protocol, completion of follow-up questionnaires no more than 7 days before the expected due date of follow-up and no more than 28 days after the expected due date of follow-up, on treatment effect for the primary outcome. Treatment effect heterogeneity by LTC will be explored but significance tests within LTC will not be presented due to the study not being powered to undertake subgroup analysis. Sensitivity analysis will be conducted for the primary outcome to assess the impact of missing data, using a pattern-mixture modelling approach, whereby models are run under a range of plausible scenarios with missing data imputed.

For moderator analysis, exploratory analysis will examine treatment effect heterogeneity for several key baseline variables that are anticipated to be potentially predictive of the treatment effect on the primary outcome. These variables include the baseline level of the primary outcome (PHQ-ADS total score), LTC (eg, chronic kidney disease, inflammatory bowel disease, multiple sclerosis and psoriasis), age (as a continuous variable) and ethnicity (black, asian and minority ethnic (BAME) vs non-BAME). Analysis for each putative moderator will include the main effect and a treatment group by moderator interaction term in the mixed-effects model used to estimate the treatment effect for the primary outcome, based on the intention-to-treat sample. Moderator analyses will be exploratory and will be published in a secondary paper since we do not specifically power for tests of effect modification.

For mediation analysis, the mechanisms of action of the treatment will be examined using mediator analysis in a structural equation modelling framework using the intention-to-treat sample. Specifically, the mediatory role of knowledge and confidence for illness self-management (PAM) on distress (PHQ-ADS) will be assessed. The mediator measured at 6 weeks and 12 weeks will be 
used to explain any treatment effects at 12 weeks. Given the contemporaneous assessment of the mediator and outcome at 12 weeks, the ability to draw conclusions about causality is limited and would be based on the consistency of the effects observed for both analyses considering mediator at both 6 weeks and 12 weeks. Analysis will estimate the total effect, indirect effect and proportion of the treatment effect on the primary outcome (PHQ-ADS total score) that occurs via this putative mediator variable identified as a target of the treatment.

\section{Economic evaluation}

Additional funding is being sought to conduct a health economic evaluation comparing COMPASS with SCS alone to assess the cost effectiveness of the treatment. If successful, health outcomes over the course of the study will be evaluated using EQ-5D-3L data and these will be extrapolated over time using various scenarios to assess the potential impact of the treatment on health-related quality of life. The resources required for delivering COMPASS will be taken from the study and combined with published cost information to ascertain the cost of delivering the treatment. All other costs relating to distress will be captured based on information from the published literature and national cost databases by linking clinical or patient-reported outcomes to resource usage. The analysis will primarily take an NHS and personal social services perspective. Results of the model will be presented at time points up to 2 years. The key outcome from the analysis will be the incremental cost per quality of life years gained. Scenario and sensitivity analyses will be carried out to assess the robustness of the cost-effectiveness results.

This analysis will be delivered by University of York Health Economics Consortium who have provided input on this protocol.

\section{Nested qualitative study}

We will conduct semi-structured qualitative interviews over the telephone or using digital interfaces (eg, teams) with a subset of participants in the COMPASS arm at two time points: (a) prior to COMPASS registration to explore participants' understanding of how their distress and LTC are linked and their expectations of the programme and (b) after end-of-treatment questionnaires have been completed to assess the acceptability of COMPASS and gather feedback about the programme. For the preCOMPASS interviews, we do not want to delay allocation to treatment after randomisation, so aim to conduct preCOMPASS interviews the day after randomisation. Across the recruitment period, we estimate that we will be able to interview 20-30 participants across all LTCs. This would represent $20 \%-30 \%$ of the COMPASS group.

For the post-COMPASS interviews, we will aim to interview 10-15 COMPASS participants for each LTC. This represents approximately $50 \%$ of the participants allocated to COMPASS from each LTC and will be feasible given participants will finish the programme and end-of-treatment questionnaires in a staggered manner, given the varying entry dates into the study.

We will purposively sample interview participants to include a range of people of different ages, ethnicities, LTCs and engagement (ie, online modules completed and phone appointments attended), to ensure implications and future improvements to the COMPASS programme are relevant across different demographic groups. Those who withdraw or drop-out in the middle of the treatment will also be interviewed if they consent to this.

All consenting therapists will be interviewed once they have finished delivering COMPASS, to assess acceptability of COMPASS and gather feedback from their user perspectives.

Interviews will be organised and conducted by members of the research team who are not involved in statistical analysis to ensure that those who are involved remain blinded to allocation. Interviews will be conducted after end-of-treatment questionnaires have been completed to avoid any contamination. In the case of participants who have withdrawn from COMPASS, they will be interviewed following their withdrawal if they consent to this, to gather feedback in a timely manner.

\section{Qualitative analyses}

Qualitative analyses will follow the Braun and Clarke's method for thematic analysis. ${ }^{44}$ Data collection and analysis occurs concurrently, so that findings raised in early interviews can be explored in subsequent ones. Transcripts will be coded line by line and once initial codes are identified they will be grouped into potential themes. Coding will occur iteratively and early identified themes will be discussed among the research team for validity until a thematic map is identified. We will undertake independent coding and cross-checking. A proportion of data will be analysed collectively in 'data clinics', where the research team will share and exchange interpretations of key themes identified from the data. The focus of analysis will be to also explore differences/nuances as well as similarities in treatment experiences across LTCs through constant comparative methodology and the framework method. Analysis will occur separately for the pre-COMPASS and post-COMPASS (after completion of the 12-week follow-up questionnaire) interviews.

\section{Research governance}

The trial will be conducted in accordance with the General Data Protection Regulation 2016 (GDPR) and MRC good clinical practice guidance in clinical trials, and other regulatory requirements as appropriate. The final trial publication will include the items recommended under the extended CONSORT statement for RCTs of non-pharmacologic interventions. ${ }^{23}$

\section{Monitoring and audit}

The trial management team will meet weekly to monitor the trial conduct and recruitment. Frequent contact via Teams and email will ensure the trial runs smoothly in 
line with the protocol. The core team includes the principal investigator, COMPASS project lead, statistical lead, research assistant and clinical supervisor. An external trial statistician will cross-check the SAP.

\section{Safety}

Risk to participants is expected to be low. COMPASS is a non-invasive talking therapy based on a CBT approach that has been extensively used across primary mental health conditions, as well as in the context of adjustment, symptom management, and comorbid anxiety and/or depression in LTCs. In the implementation feasibility work prior to this trial, none of the participants raised concerns about the content of the treatment being too distressing.

Suicidal risk will be assessed during eligibility screening using PHQ-9 item 9. However, people who take part in this study will be experiencing distress, by the very nature of what COMPASS aims to treat. One of the outcome measures (PHQ-9) also specifically asks whether someone has 'been bothered by feeling like they would be better off dead or hurting themselves in some way' in the last 2 weeks. Therefore, it is imperative that a robust risk management system is in place, both for therapists who are providing the guided support and for the research team members who are conducting the qualitative interviews.

Standard operating procedures (see below) will be enacted in response to evidence of risk of recent selfharm or current intent.

The following principles and procedures govern risk assessment and reporting.

- General procedures.

Whenever any significant risk is identified, a risk assessment should be completed and (counter signed) by the principal investigator and/or nominated deputy as soon after the assessment as possible.

Any significant, but not imminent risk, should be reported to the person's GP and, if appropriate, other healthcare professionals, as soon as is reasonably possible.

Any imminent risk should lead to the immediate involvement of the appropriate emergency health services. The principal investigator must ensure that research associates, assistants and students are fully informed and competent to follow the procedures.

When the principal investigator is away, they should ensure appropriate cover is arranged for any risk issues that might arise in their absence.

\section{- Exploring risk.}

There are seven questions to be used following any indication of risk from responses to interview questions or any other sources. We define risk as any reporting active plans of suicide/self-harm.

Ask the Exploring Risk in Research Interviews questions and then look at answers from the sheet to determine the level of risk: A (low), B or C (high).

The risk assessment script and related actions should be performed when:
- Active suicidal plans are disclosed during a patient interview.

OR

- The patient indicates the below response on item 9 on the PHQ-9 and associated follow-up risk questions during their 6-month follow-up questionnaire.

- PHQ-9: item 9 score of 1 or more. AND

- Answers 'Yes' to extra risk question: 'are you currently making plans about how you would end your life?'.

\section{Risk management}

Managing risk will be covered in the therapist and researcher (screening and qualitative interviewing) training. A 'standard operating procedure for risk' document outlines steps required to conduct a risk assessment. This includes a series of questions to explore risk with the individual, covering plans, actions and prevention/protective factors, and subsequent actions required depending on patient's responses.

As the COMPASS programme also sends questionnaires for patients to answer in context of the treatment (ie, to guide the support calls and provide the therapist with markers of progress), the COMPASS programme also triggers a risk flow. This occurs if the patient scores 1 or more on item 9 of the PHQ-9 questionnaire AND answers 'Yes' to Q1 of the additional risk questions: 'are you currently making plans about how you would end your life?'. In this instance, the therapist receives an email, a designated 'risk buddy' (research study email address) also receives this email in case the therapist is away, the patient is moved up the therapist caseload and an icon is highlighted to flag the risk.

A member of the research team will monitor baseline, mid-treatment and end-of-treatment PHQ-9 outcome responses, and follow-up to conduct a risk assessment with any individuals who meet the criteria described above. If necessary, a referral will be made to ensure the patient gets the support they need.

\section{Adverse and serious adverse events}

Adverse events (AEs) are defined as any clinical change, disease or disorder that participants experience during their involvement in the trial. AEs can be both related and unrelated to trial participation.

A serious adverse event (SAE) is classified as an $\mathrm{AE}$ that results in death, threat to life, in-patient hospitalisation, disability/incapacity, congenital anomaly or birth defect or any other medical event requiring intervention to prevent one of these. If the patient has a planned hospitalisation, this will not be regarded as an AE.

AEs and SAEs raised during the trial will be documented by $\mathrm{KH}$ and discussed with the clinical lead and/ or principal investigator at trial meetings. If the SAE is deemed to be a consequence of or reaction to participation in the trial this will be documented and reported to the Ethics Committee. A decision will be made among the trial team, and COMPASS guide if applicable, whether 
the participant should be withdrawn from the treatment and research trial. SAEs will be reviewed after 1 month, and on an on-going basis if not resolved.

All participants will also be asked to report AEs at the end-of-treatment questionnaire based on guidelines for standard reporting of adverse events in clinical trials. ${ }^{45}$ This was adapted to include any AEs-related personal circumstances, in line with recommendations for reporting of adverse events in trials of psychological interventions. ${ }^{46}$ Deterioration on the primary outcome measure (PHQ-ADS) will also be classed as an AE, specifically an increase in 6 on PHQ and 4 on GAD, in line with IAPT's 'reliable deterioration' classification. ${ }^{47}$ In relation to life-threatening events, unplanned hospitalisations and events resulting in permanent disability/incapacity, participants will also be asked if these events are related to their physical or mental health.

\section{Stopping rules}

Protocol deviations, changes to treatment and breaking of the randomisation code will only occur after deliberation by the trial team if it becomes apparent that the active treatment (COMPASS) is causing a consistent pattern of deterioration, or if there is another obvious and significant clinical necessity.

\section{Data protection and storage}

Any identifiable or sensitive data will be kept in passwordprotected files on secure KCL drives, in a folder only accessible to members of the research team with designated clearance. Participants will be allocated a sevendigit study ID number, randomly generated by Qualtrics, which will be used for analysis processes. Qualtrics provides technology to store data securely online that is GDPR compliant and is, therefore, suitable for the storage of sensitive data.

Participants who are allocated to and register with COMPASS will also be assigned a COMPASS ID number by the programme. Any correspondence with therapists about participants (eg, informing them of someone being allocated to their caseload) will only use this ID number and first and last initials. No identifiable information will be shared.

COMPASS collects data automatically in a pseudoanonymised format. The pseudo-anonymised ID number that links to the patient identifiable information will be recorded in the password-protected participant masterfile. Members of the team involved in statistical analysis will not have access to this file. This information will be downloaded at the end of the study and stored in a password protected file on a KCL server. The COMPASS website is hosted in compliance with NHS hosting requirements and the suppliers (SPIKA) are NHS IG Toolkit approved.

Qualitative interviews will be audio-recorded using an encrypted device and transcribed verbatim by either the research team or professional transcription service. Files will be transferred using KCL's secure server. Any transcription company will be experienced at dealing with confidential data (including from NHS patients). The participant's full name will never be used during the interview and all participants will be assigned a pseudonym when data are presented. Once the data have been transcribed, the transcription will be crosschecked with the recording and then all audio-recordings will be destroyed. Any identifiable information will also be omitted from the transcripts. Only anonymised transcribed data will be retained and stored on a password-protected file on a networked KCL computer.

Therapy sessions will be recorded for training and supervision purposes. The participant will be informed beforehand and will be able to opt out of having their therapy session recorded. The sessions will be recorded using an encrypted device and stored securely on the KCL server. Transfer, if necessary (for supervision or fidelity purposes), will occur either via secure nhs.net to nhs.net email or using the secure KCL file transfer service.

Screening questionnaires and consent forms will be completed electronically via Qualtrics. This information will be stored in the system, which can only be accessed via a password-protected account. Personal data (email addresses which are required to complete the questionnaire online using REDCap survey software) will be stored in the Qualtrics survey software, which requires the study-specific account and password to access. Questionnaire data will be collected via REDCap, but no personal identifiable information will be stored on REDCap.

The raw data will be kept according to KCL's data management policy. According to this policy, the pseudoanonymised raw data will be stored securely at KCL for 7 years following the end of study and then destroyed securely.

All data will be managed in line with General Data Protection Act (2018) and Caldicott principles. Confidentiality will only be breached if there is a severe threat to the patient or others. The study chief investigator remains responsible for overseeing data collection, quality and recording.

The data that support the findings of this study will be available on request from the corresponding author. The data will not be publicly available due to privacy or ethical restrictions.

\section{Patient and public involvement}

Patient and public involvement (PPI) and taking a personcentred approach are key elements of research and intervention development ${ }^{48} 49$ and occurred throughout the COMPASS development process. Patient representatives have been included at all stages, including conceptualisation and naming of the programme, content feedback, healthcare integration planning, patient information leaflets/website creation and participant material creation for research. In total, over 30 patient representatives are part of the COMPASS PPI advisory group. Long-term conditions represented include inflammatory bowel disease, endometriosis, cancer, multiple sclerosis, sarcoidosis, rheumatoid arthritis, kidney disease, diabetes and 
asthma. Our PPI group was recruited via adverts placed within our local partner NHS trusts.

Healthcare practitioners (including IAPT's psychological well-being practitioners and clinical psychologists) have also been involved in developing the programme and helped to optimise training and implementation, for example, designing the therapist platform, identifying training needs and identifying enhancements to improve usability. As with patients, small group meetings, individual interviews and ThinkAloud methods were used to gather feedback.

This involvement has been imperative to ensure the software, functionality and usability of the programme is suited to the intended use (treating illness-related distress) and identified patient population (people with LTCs).

For this research study specifically, we gathered input from 11 patient representatives (June 2020) on the lay overview of the research $(n=4)$, resources that are accessed for support and ideas for recruitment avenues $(\mathrm{n}=9)$.

Moving forward, we will seek advice from representatives on how to disseminate findings across patient networks. Once the research is completed, we will send an update to participants and PPI members to inform them of the findings.

\section{ETHICS AND DISSEMINATION}

\section{Ethical approval}

This study has been reviewed by King's College London Research Ethics Committee because recruitment will be from UK charities and not NHS sites (reference: LRS-19/20-20347). Any protocol modifications will be discussed within the research team and explained to the Ethics Committee if applicable. If modifications impact on treatment provision, participants will be informed in writing by email.

COMPASS has been developed under the control of the Kings Digital Therapies Quality Management System in compliance with the Medical Devices Directive 93/42/ EEC for class I medical devices and, therefore, all clinical safety and quality management protocols are in place and are adhered to. We have an independent digital clinical safety officer who oversees this.

\section{Informed consent}

If participants are deemed eligible after the screening phone call, they will be sent the participant information again and a link to an online consent form, hosted on Qualtrics. All participants will provide informed consent to take part, by agreeing with the given statements and providing an online signature, before they enter the study and complete baseline measures.

\section{Dissemination}

Findings will be published in peer-reviewed journals and presented at national and international conferences. We will disseminate findings to patients and professionals throughout the UK through our partner charities, via their websites, social media and other communication outputs. Our patient advisory group will help with press releases and lay presentations. During the trial, we will use social media communications to provide regular updates to participants.

\section{Trial status}

Recruitment started in late November 2020 and ended in March 2021. Data collection was completed in July 2021 and data analysis is currently underway, due to complete in October 2021.

\section{DISCUSSION}

This protocol details an RCT to evaluate the efficacy of COMPASS, a newly developed, therapist-supported, digital CBT programme designed to treat distress (anxiety and depression) in the context of LTCs. A significant strength of the trial is the ability to conduct it during the COVID-19 pandemic. We can provide participants with psychological support whilst not placing burden on overstretched NHS services. The study also uses a novel 'hub' delivery model, through delivering care remotely to people recruited via national LTC charities, by using the COMPASS web programme and trained therapists working remotely as part of the COMPASS team. Additionally, when completed, this will be the first trial of a transdiagnostic digital programme designed specifically to treat LTC-related distress. A limitation of the study is that it is not possible to standardise the charity support arm. The different charities offer a variety of options for support. The control condition is also not matched for time and attention of the therapists, although this is minimal (3 hours maximum per patient). Additionally, the COMPASS therapists will have different levels of experience. All will have a clinical or health psychology background and CBT training, but some will have worked in the health psychology field longer than others. COMPASS training, regular supervision and fidelity checking aim to keep the guided support as consistent and high quality as possible, to mitigate against therapist effects. As part of the COMPASS treatment, participants are asked to complete mood measures for therapeutic feedback at two points in the programme. The extra measurement in the one arm may introduce bias, but this is likely to be small. Finally, another limitation to consider is that participants are selfselected volunteers. The results of the trial will inform modifications necessary to further enhance COMPASS. In the context of LTCs, patients may face relapses and illness progression/disability, so evaluating how sustainable any improvements in distress following COMPASS are using longer follow-up is, therefore, essential.

Twitter Katrin Hulme @katrinhulme and Federica Picariello @fede_picariello

Contributors Authorship criteria: substantial contribution to conceptualisation or design of study/data analysis/study implementation, drafting or revising 
protocol and final approval of the publication. KH: conceptualisation, design, study implementation and draft writing/revising. JLH: conceptualisation, design and draft writing/revising. FP: design, statistical analysis, study implementation and draft writing/revising. NS: design, qualitative analysis, study implementation and draft writing/revising. SN: statistical analysis and revising. AW - design, study implementation and revising. RM-M: conceptualisation, design, study oversight and revising.

Funding This work was supported by King's Health Partners Mind Body Programme and NIHR Maudsley Biomedical Research Centre.

Competing interests None declared.

Patient consent for publication Not applicable.

Provenance and peer review Not commissioned; externally peer reviewed.

Supplemental material This content has been supplied by the author(s). It has not been vetted by BMJ Publishing Group Limited (BMJ) and may not have been peer-reviewed. Any opinions or recommendations discussed are solely those of the author(s) and are not endorsed by BMJ. BMJ disclaims all liability and responsibility arising from any reliance placed on the content. Where the content includes any translated material, BMJ does not warrant the accuracy and reliability of the translations (including but not limited to local regulations, clinical guidelines, terminology, drug names and drug dosages), and is not responsible for any error and/or omissions arising from translation and adaptation or otherwise.

Open access This is an open access article distributed in accordance with the Creative Commons Attribution Non Commercial (CC BY-NC 4.0) license, which permits others to distribute, remix, adapt, build upon this work non-commercially, and license their derivative works on different terms, provided the original work is properly cited, appropriate credit is given, any changes made indicated, and the use is non-commercial. See: http://creativecommons.org/licenses/by-nc/4.0/.

\section{ORCID iDs}

Katrin Hulme http://orcid.org/0000-0002-2623-4417

Federica Picariello http://orcid.org/0000-0002-2532-3290

\section{REFERENCES}

1 Naylor C, Parsonage M, McDaid D, et al. Long-Term conditions and mental health: the cost of co-morbidities, 2012. Available: https:// www.kingsfund.org.uk/sites/default/files/field/field_publication_file/ long-term-conditions-mental-health-cost-comorbidities-naylor-feb12. pdf

2 Hutter N, Schnurr A, Baumeister H. Healthcare costs in patients with diabetes mellitus and comorbid mental disorders--a systematic review. Diabetologia 2010;53:2470-9.

3 Naylor C, Das P, Ross S, et al. Bringing together physical and mental health. King's Fund, 2016. Available: http://www.kingsfund.org.uk/ sites/files/kf/field/field_publication_file/Bringing-together-KingsFund-March-2016_1.pdf

4 Wakefield S, Kellett S, Simmonds-Buckley M, et al. Improving access to psychological therapies (IAPT) in the United Kingdom: a systematic review and meta-analysis of 10-years of practice-based evidence. Br J Clin Psychol 2021;60:1-37.

5 Catarino A, Bateup S, Tablan V, et al. Demographic and clinical predictors of response to internet-enabled cognitive-behavioural therapy for depression and anxiety. BJPsych Open 2018;4:411-8.

6 De Lusignan S, Jones S, McCrae N. IAPT LTC/MUS Pathfinder evaluation project. Surrey, UK: University of Surrey, 2016.

7 Nobis S, Lehr D, Ebert DD, et al. Efficacy of a web-based intervention with mobile phone support in treating depressive symptoms in adults with type 1 and type 2 diabetes: a randomized controlled trial. Diabetes Care 2015;38:776-83.

8 Moussavi S, Chatterji S, Verdes E, et al. Depression, chronic diseases, and decrements in health: results from the world health surveys. Lancet 2007;370:851-8.

9 Hudson JL, Moss-Morris R. Treating illness distress in chronic illness. Eur Psychol 2019;24:26-37.

10 Moss-Morris R. Adjusting to chronic illness: time for a unified theory. Br J Health Psychol 2013;18:681-6.

11 Murray E, Hekler EB, Andersson G, et al. Evaluating digital health interventions: key questions and approaches. Am J Prev Med 2016;51:843-51.

12 May C, Gask L, Atkinson T, et al. Resisting and promoting new technologies in clinical practice: the case of telepsychiatry. Soc Sci Med 2001;52:1889-901.
13 Alvidrez J, Azocar F. Distressed women's clinic patients: preferences for mental health treatments and perceived obstacles. Gen Hosp Psychiatry 1999;21:340-7.

14 Hollon SD, Muñoz RF, Barlow DH, et al. Psychosocial intervention development for the prevention and treatment of depression: promoting innovation and increasing access. Biol Psychiatry 2002;52:610-30.

15 Nutting PA, Rost K, Dickinson M, et al. Barriers to initiating depression treatment in primary care practice. J Gen Intern Med 2002;17:103-11.

16 Andersson G, Cuijpers P, Carlbring P, et al. Guided Internet-based vs. face-to-face cognitive behavior therapy for psychiatric and somatic disorders: a systematic review and meta-analysis. World Psychiatry 2014;13:288-95.

17 Karyotaki E, Efthimiou O, Miguel C, et al. Internet-Based cognitive behavioral therapy for depression: a systematic review and individual patient data network meta-analysis. JAMA Psychiatry 2021;78:361-71.

18 Holmes EA, O'Connor RC, Perry VH, et al. Multidisciplinary research priorities for the COVID-19 pandemic: a call for action for mental health science. Lancet Psychiatry 2020;7:547-60.

19 Kidney Care UK. Worried sick - the impact of COVID-19 on people living with kidney disease: findings from a patient survey, 2020.

20 Kroenke K, Wu J, Yu Z, et al. Patient health questionnaire anxiety and depression scale: initial validation in three clinical trials. Psychosom Med 2016;78:716-27.

21 Loudon K, Treweek S, Sullivan F, et al. The PRECIS-2 tool: designing trials that are fit for purpose. BMJ 2015;350:h2147.

22 Kroenke K, Spitzer RL, Williams JBW, et al. An ultra-brief screening scale for anxiety and depression: the PHQ-4. Psychosomatics 2009:50:613-21.

23 Moher D, Hopewell S, Schulz KF, et al. Consort 2010 explanation and elaboration: updated guidelines for reporting parallel group randomised trials. BMJ 2010;340:c869.

24 Richards DA. Clinical Case Management Supervision. In: The Wiley international Handbook of clinical supervision. John Wiley \& Sons, Ltd, 2014: 518-29.

25 French DP, Miles LM, Elbourne D, et al. Reducing bias in trials from reactions to measurement: the merit study including developmental work and expert workshop. Health Technol Assess 2021:25:1-72.

26 National Collaborating Centre for Mental Health. The improving access to psychological therapies (IAPT) pathway for people with long-term physical health conditions and medically unexplained symptoms: full implementation guidance. London: NICE, 2018.

27 Everitt H, Landau S, Little P, et al. Assessing cognitive behavioural therapy in irritable bowel (ACTIB): protocol for a randomised controlled trial of clinical-effectiveness and cost-effectiveness of therapist delivered cognitive behavioural therapy and web-based self-management in irritable bowel syndrome in adults. BMJ Open 2015;5:e008622.

28 Everitt HA, Landau S, O'Reilly G, et al. Assessing telephonedelivered cognitive-behavioural therapy (CBT) and web-delivered CBT versus treatment as usual in irritable bowel syndrome (ACTIB): a multicentre randomised trial. Gut 2019;68:1613.

29 Kroenke K, Spitzer RL. The PHQ-9: a new depression diagnostic and severity measure. Psychiatr Ann 2002;32:509-15.

30 Spitzer RL, Kroenke K, Williams JBW, et al. A brief measure for assessing generalized anxiety disorder: the GAD-7. Arch Intern Med 2006;166:1092-7.

31 Mundt JC, Marks IM, Shear MK, et al. The work and social adjustment scale: a simple measure of impairment in functioning. $\mathrm{Br}$ $J$ Psychiatry 2002;180:461-4.

32 Moss-Morris R, Weinman J, Petrie K, et al. The revised illness perception questionnaire (IPQ-R). Psychol Health 2002;17:1-16.

33 Rabin R, de Charro F, Fd C. EQ-5D: a measure of health status from the EuroQol group. Ann Med 2001;33:337-43.

34 Hibbard JH, Mahoney ER, Stockard J, et al. Development and testing of a short form of the patient activation measure. Health Serv Res 2005;40:1918-30.

35 Guy W. Clinical global impression scale. The ECDEU assessment manual for psychopharmacology. Rockville, MD: National Institute of Mental Health, 1976: 218-22.

36 Yalcin I, Bump RC. Validation of two global impression questionnaires for incontinence. Am J Obstet Gynecol 2003;189:98-101.

37 Mc Sharry J, Bishop FL, Moss-Morris R, et al. A new measure of multimorbid illness and treatment representations: the example of diabetes and depression. J Affect Disord 2015;174:192-200.

38 Thornicroft G. An adapted version of the Client Service Receipt Inventory. In: Beecham J, Knapp M, eds. Costing psychiatric interventions. London: Measuring Mental Health Needs, 2001. 
39 National Collaborating Centre for Mental Health. Depression in adults with a chronic physical health problem: treatment and management. London: The British Psychological Society and The Royal College of Psychiatrists, 2010.

40 Picariello F, Moss-Morris R, Norton S, et al. Feasibility trial of cognitive behavioral therapy for fatigue in hemodialysis (BReF intervention). J Pain Symptom Manage 2021;61:1234-46.

41 Scott W, Guildford BJ, Badenoch J, et al. Feasibility randomizedcontrolled trial of online acceptance and commitment therapy for painful peripheral neuropathy in people living with HIV: the open study. Eur J Pain 2021;25:1493-507.

42 Hudson JL, Moss-Morris R, Norton S, et al. Tailored online cognitive behavioural therapy with or without therapist support calls to target psychological distress in adults receiving haemodialysis: a feasibility randomised controlled trial. J Psychosom Res 2017;102:61-70.

43 Bogosian A, Hughes A, Norton S, et al. Potential treatment mechanisms in a mindfulness-based intervention for people with progressive multiple sclerosis. Br J Health Psychol 2016;21:859-80.
44 Braun V, Clarke V. Using thematic analysis in psychology. Qual Res Psychol 2006;3:77-101.

45 loannidis JPA, Evans SJW, Gøtzsche PC, et al. Better reporting of harms in randomized trials: an extension of the CONSORT statement. Ann Intern Med 2004;141:781-8.

46 Duggan C, Parry G, McMurran M, et al. The recording of adverse events from psychological treatments in clinical trials: evidence from a review of NIHR-funded trials. Trials 2014;15:1-9.

47 Clark D, Oates M. Improving access to psychological therapies: measuring improvement and recovery adult services. London: NHS England, 2014.

48 O'Cathain A, Croot L, Duncan E, et al. Guidance on how to develop complex interventions to improve health and healthcare. BMJ Open 2019;9:e029954.

49 Yardley L, Morrison L, Bradbury K, et al. The person-based approach to intervention development: application to digital health-related behavior change interventions. J Med Internet Res 2015;17:e30. 